\title{
A Model of Media Information Management for Content Retrieval
}

\author{
Lin Liu \\ The Department Of Computer Science, Chongqing College of Electronic Engineering, \\ Chongqing 401331, China \\ liulin1402@163.com
}

\begin{abstract}
The rapid development of digital library makes the multimedia informattign resources organization and management become a key issue to develdp the difital library, and the multimedia information retrieval technology has become the core technology of the digital book data management, the existing multimedia retrieval model cannot effectively implement multimedia digital books data management. Therefore, this paper proposes a content-based multimedia retrieval data model and combined with the differen modal data abstraction and decomposition classification thought, discusses the significance of this data model, also briefly introduces the building method of this model. Finally by combining this design it implements a multimedia digital books dotaretrieval s.85tgm.
\end{abstract}

Keywords: multimedia data mode1, digital library multimedia data management, content retrieval

\section{Introduction}

Digital libraries, evolved from traditional libraries during the information age, have become an important platform for information retrieval [1]. However, following the rapid expansion of multimedia data in the collection of digital libraries, such as text, images, audio and video data, traditional information retrieval approaches became inadequate to the management of rarge unstructuded data. Firstly, it is insufficient for the support of interaction with multimedia data. Users are usually passive roles only provided with one-way access to multimedia data. Even though multimedia data carry a rich amount of information, it still has difficulty to acknowledge in systematic management of such unstructured data. Secondly, existing models that associate multimedia data with the keywords are for information managementand retrieval, somehow multimedia data are often stored as object attributes with low granularity, which is prone to cause data redundancy and can hardly support content-level information retrieval. Besides, the distributed structure which is considered as one of the optimal solutions for developing search engines [2-3], and with advantages in information retrieval from massive data, is also facing a big challenge in the efficient organization and management of multimedia data.

Considered to digital libraries, the effective approach to manage multimedia data is to automatically handle the embedded information in its content. As the traditional libraries, users can find aimed objects from the multimedia by using indexing tools like cards. By analogy with that, a structured index can also be established for the content of multimedia data in digital libraries, so that the embedded information can be exposed for retrieval, and unordered multimedia data can be translated into index-based data which is more suitable for storage and access control with fine granularity [4-5]. 
Therefore, there is a critical need for proper data models to organize and manage massive multimedia data, which should achieve to provide multimedia information retrieval service for digital libraries by means of multi-level semantic data mining. According to such needs, this paper proposes an efficient data model for multimedia content retrieval. Section 2 presents the detail of our data model.

\section{Related Work}

\subsection{The Multimedia Data Modeling}

Compared to the ordinary multimedia data structured data is much more complicated, first the structure of multimedia data is semi-structured data (such as XML and HTMY) and unstructured (text, images, audio and video), in addition the large amounts of multimedia data content information it carries, people with different background knowledge has various understanding of the content [6]. The purpose of the organization and structured multimedia database system is for content retrieval [7]. In this sense the construction of multimedia data model is one of the key factors.

The method for modeling multimedia data, it usually can be divided into the following three kinds of modeling methods: extended relational model modeling method, the semantic model modeling method and object-oriented modeling method [8].In recent years, aimed to the multimedia database system and multimedia management has appeared a lot of data model. In [9] the algebra video (algebraic yideo) data model is one of the earliest and fairly complete data model, which has a great influence in the following models. This model can distinguish between physical streaming and logie yideo segments, and also allow users to synthesis of parallel video performance, which is provided by the support of video algebra (video algebra). However, this model is very difficult to join automatic video segmentation methods. H. Jiang proposed a video Data Model called Logical Hypervideo Data Model(LHVDM) [10] This model is able to perform multiple levels video extraction (present the video object of interest to the users), it is referred to as hot objects, and also it put the extracted semantic and other logic extraction together(including other hot objects). DISIMA [11] is the image database system developed by the university of Alberta, which proposes a model applicable to image and space applications. The generic indexing model - VIDEX is proposed in [12], that is applied to the multimedia information system SMOOTH [13].This method combines the advantages introduced by [9-10], introduces the video streams structured approach and indexing process versatility extends in these models. Farham Mohamed [14] proposed a data model based on temporal to manage the multimedia data. Besides Liv presented a multi-media data organization and management model which based on object-oriented [15].

Thought the current multimedia data model solved the related problems about modeling a single media, many different media integrated modeling, the correlation analysis between different media data and seamless integration in digital library still did not perform well. Object-oriented method provides an expression, management, and share options for multimedia data modeling scheme. However, a monomedia oriented object model is very difficult to meet the needs of the digital library, which is in the demand of a variety of media data organization and management. Digital library needs to deal with a variety of types media data, and these different types of media data for unified modeling and management, therefore need a more powerful multimedia data model to support effective modeling and seamless integration with digital library application demand, realize all levels semantic mining of the multimedia data and provide efficient digital book data retrieval service. 


\subsection{Multimedia Content Analysis and Classification}

Multimedia data contain rich semantic content information, which mining and extract the semantic information from, lead to more efficiently manage and utilized the multimedia data, also acquire effective multimedia data management model.The accurate understanding and analysis of multimedia content and favourable classification are the basis of structuring content-based multimedia data model, and it always is a research focus in the field of multimedia data management and retrieval.Torresani [16] by learning a concise image descriptors to achieve the effective identified the unknown scene class. Li [17] proposed a multi-resolution representation model to classify the scenes. Recent years, scholars through the low cost of crowdsourcing and intelligent human-machine interaction technorogy)[18], provides a series description of semantic information and the visual consistency to the mages, and constructs low-level visual features through the statistical learning model, lead to establish the mapping relationship between the visual properties and category labels (19-21), provides an effective solution for image recognition. J.A nibar Arias [22] proposed a VUDLA model, which is the support of multimedia file storage, indexing, and comments.

The biggest challenge multimedia digital books retrieval system faced is how to make up for the semantic gap, based on the content analysis and classification of annotation driven intelligent processing method has become one of the most promising solution. Despite the current multimedia data model has solved the single media content analysis and classification for the related problems, but many different media integrated modeling and multimedia data, which based on the associated contenteanalysis and nultimedia digital books data retrieval system seamlessly integrate, do not(perform well. Muitimedia content analysis and tagging provide an expression and management option formultimedia data. However, analysis and classification of tagging oriented the single media content are hard to meet the needs that the digital library in the demand for a variety of media data organization and management. Digital library needs to deal with a variety of different types of media data, and these different types of media data ask for unified marks and management, therefore need a more powerful multimedia data model to support effective modeling and seamless integrate with media digital book data retrieval system, that realize all levels the multimedia data semantic mining and provide efficient digital book data retrieval service.

\section{The Objective and Method of Models}

\subsection{The Objective of the Model}

The objective of our proposed data model is to allow reasonable abstraction and classification of multimedia data by decomposing each multimedia file into multiple semantic layers with well preserved content information at different levels of granularity. By using the data nodel we proposed, a multimedia information retrieval system can categorize data objects into different granular sets, so as to ensure that each semantic level (including hypermedia links) within the content of multimedia data is accessible to the information retrieval system. Moreover, in order to improve the accuracy and flexibility of information retrieval, our data model offers an interface for manual editing of properties for each data object.

\subsection{Data Abstraction Method}

The data model this paper proposed is supposed to be used for multimedia information retrieval services and the modeling targets are multimedia data. To adopt data abstraction in object-oriented methods, various multimedia forms can be modeled by different kinds of 
basic classes. Each data file is related to a source object which is an instance of the basic class. Take a complex multimedia file in the consideration; the source object can be decomposed into several atomic objects with low granularity. Each atomic object contains partial properties of the source object, and corresponds to a certain part of the source object. The data abstraction method is depicted in Figure 1.

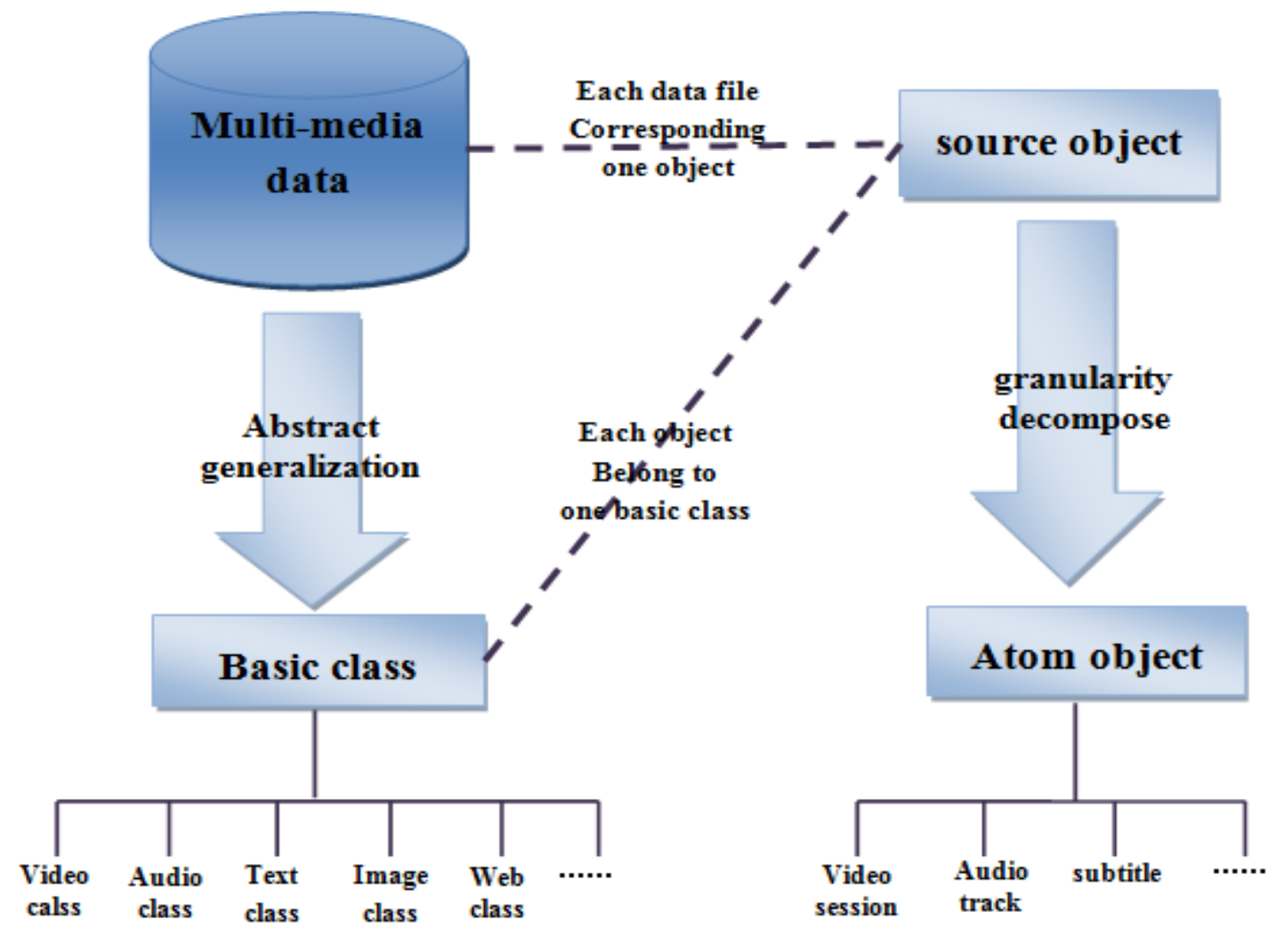

Figure 1. Data Abstraction Schematic Diagram

\subsection{Data Processing}

One source object corresponds to one data file and an atomic object corresponds to a certain part of the original data file (e.g. the audio track and subtitles of a video). Thus, all essential properties extracted from the original data file are stored in the source object including the address property of its physical location. An atomic object has an additional property indicating which part of the original data files it belongs to. Objects are mapped from the original data files, and stored in an index database, which is ready to be used for information retrieval without the concern of the original data files. We provide an editing interface for the index database, in response to the object property editing method. Figure 2 illustrates the data processing method. 


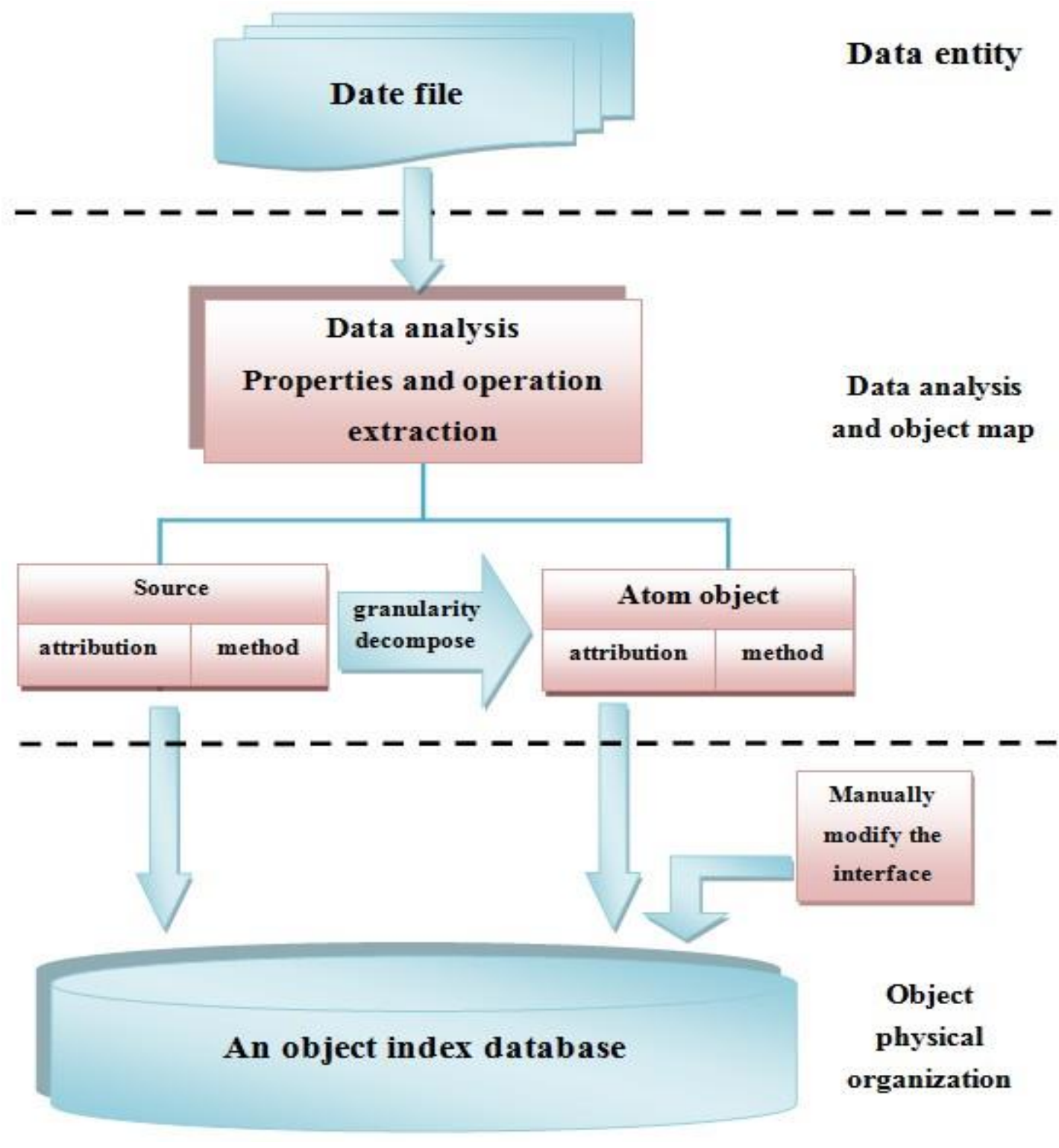

Figure 2. Data Processing Schemes Diagram

\subsection{Object Structure and Organization Method}

The common data structure for a multimedia object is <OBJ:\{ID, Type, Ti, Attr, Method $\}>$ [23], where "ID" is the unique identity of the object, "Ti" represents time attribute, and "Method" includes operations between object properties, also relations between this object and its original data file or other objects. In our data model, an object is described by a node, as showed in Figure 3, whose data structure includes the following fields:

1) Object: save its properties and method functions

2) Predecessor pointer: points to its predecessor node

3 ) Successor pointer: points to its successor node

4) Branch pointer: points to the list of its atomic objects

5) Media pointer: points to the corresponding hypermedia list of the objects 


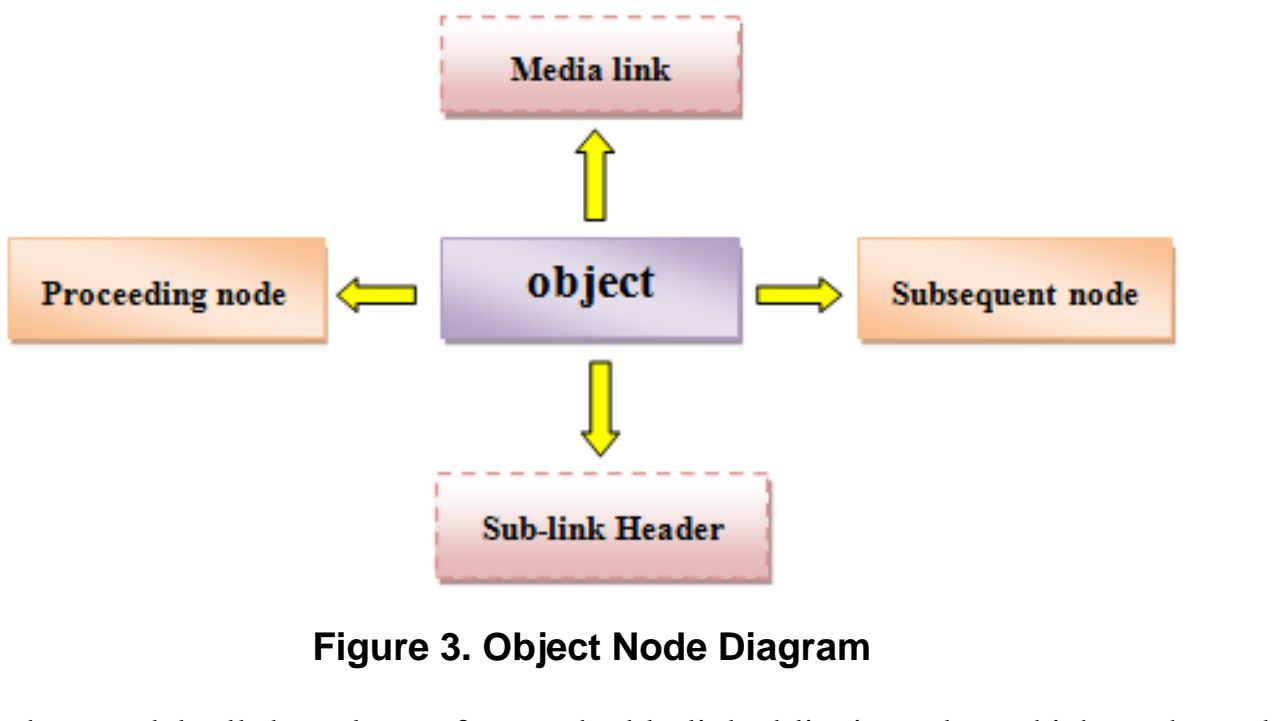

In the data model, all data classes form a doubly linked list in nodes, which as showed in Figure 4. Each node points to a header which points to another doubly linked list formed by the nodes of the same class, as showed in Figure 5.

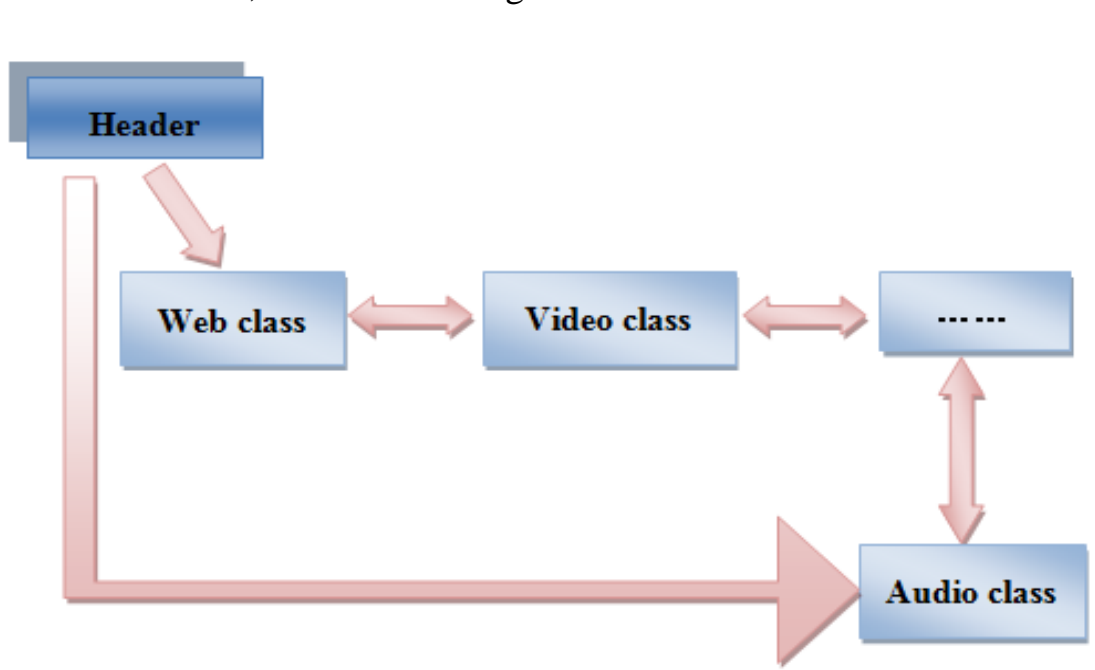

Figure 4. Two-way Linked List Class Node Diagram

Object nodes should be organized by considering the relationship between object properties, and different organization methods based on different object properties can be employed according to specific applications [23]. In practice, many data objects in a digital library can be connected by timestamp, and the same media data usually have different versions from different time. Based on such observation, the linked list of the object nodes in our data model is organized by time attribute. Thereby achieving efficient management and enabling time-based indexing. 


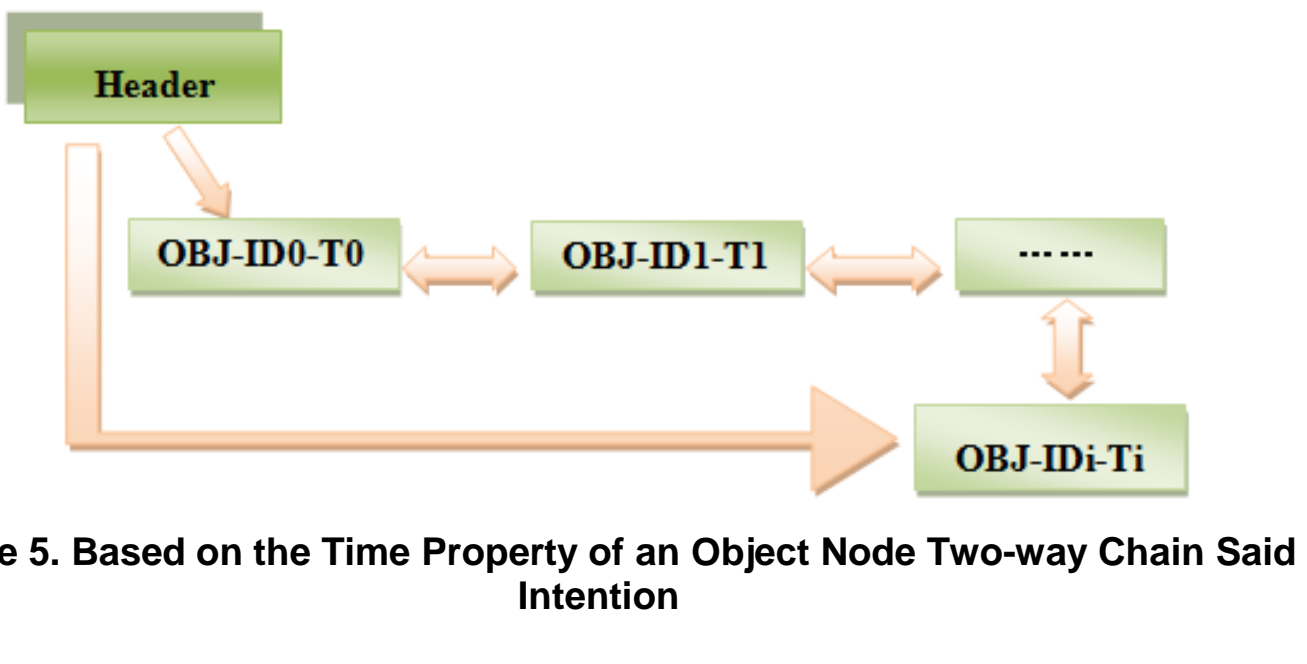

As the structure we mentioned above, a hierarchical node-object model [24] is eventually created, as showed in Figure 6. The top level is a doubly linked list of class nodes; each class node has a pointer pointing to a doubly linked list of its source objects; each source object has a pointer pointing to a doubly linked list of its atomic objects. Based on such data model, hypermedia semantics can be well preserved by using pointers instead of hypermedia links.

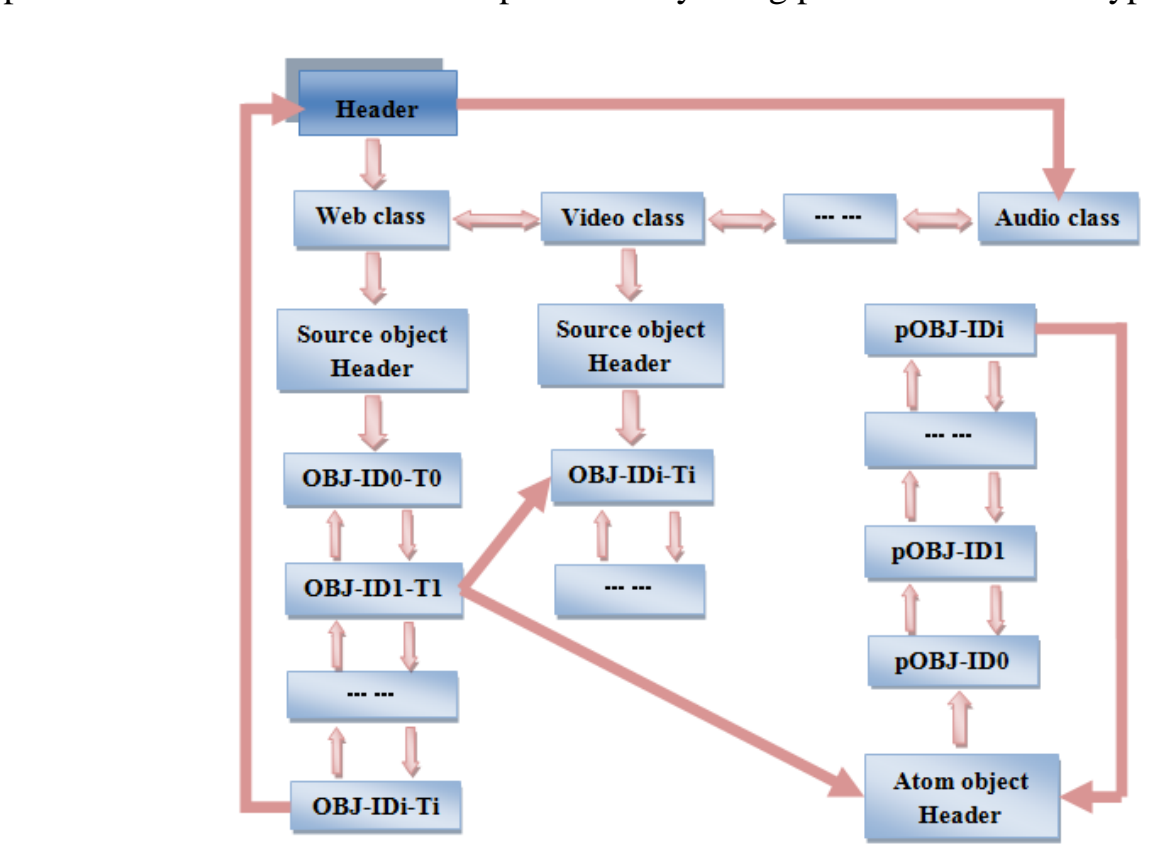

Figure 6. Model Organization Structure

\section{The Data Model Construction}

The data using object-oriented modeling requires finding the suitable object, and determining the object particle size [25], in the multi-media information retrieval system, one reasonable method for object classification is referred to the media type. Hence, all multimedia data are divided into video, audio, images, text and web pages. Wherein web and video contain plentiful content, and also include various media forms itself. Therefore, web class 
objects can be re-subdivided particle size into video, audio, images, text; video class objects subdivide particle size into audio, video and subtitles.

Data ID, data type, data time and address etc., these basic attributes of all the classes are saved in the model. Besides each object class, according to its various defined attributes and associated operation, all the data can be ultimately expressed as the object model in Figure 7.

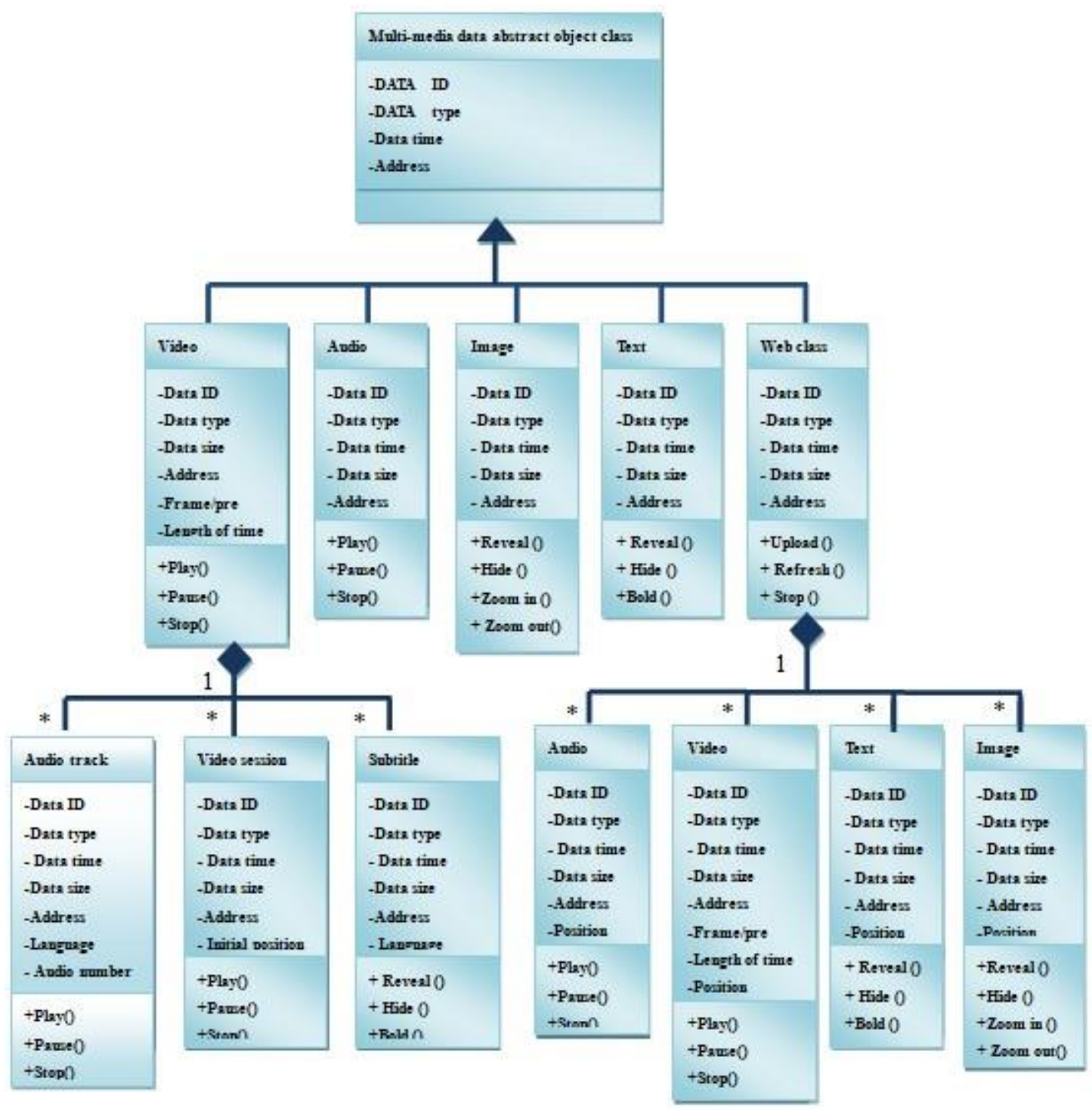

Figure 7. The Object Oriented Multimedia Retrieval Model

\section{Application Example}

The model this paper proposed can be applied to digital library multimedia data organization and management, Figure 8 based on this data model. It is an application example that uses for digital library multimedia data content retrieval and displayed.

In the index system, in the range column it chooses "all", and to retrieve the semantics of the "Yangtze river", that is to list the all file objects accorded with the semantics of the "Yangtze river" in the retrieve results column; choose the "The Yangtze river three 
gorges.avi" in the column, then the system begins to play this file; at the right side of the media link column it shows the hypermedia of this file, as the video begins to play, the "Introduction of the Yangtze river three gorgest.txt" and "SanxiaPic_1.bmp" are activated, besides revealed in the text box and photograph column of the system; the atom object column at the right side, it denotes the subset objects of this file, that means this video file can be subdivide particle size into audio, video session, subtitles and so on.

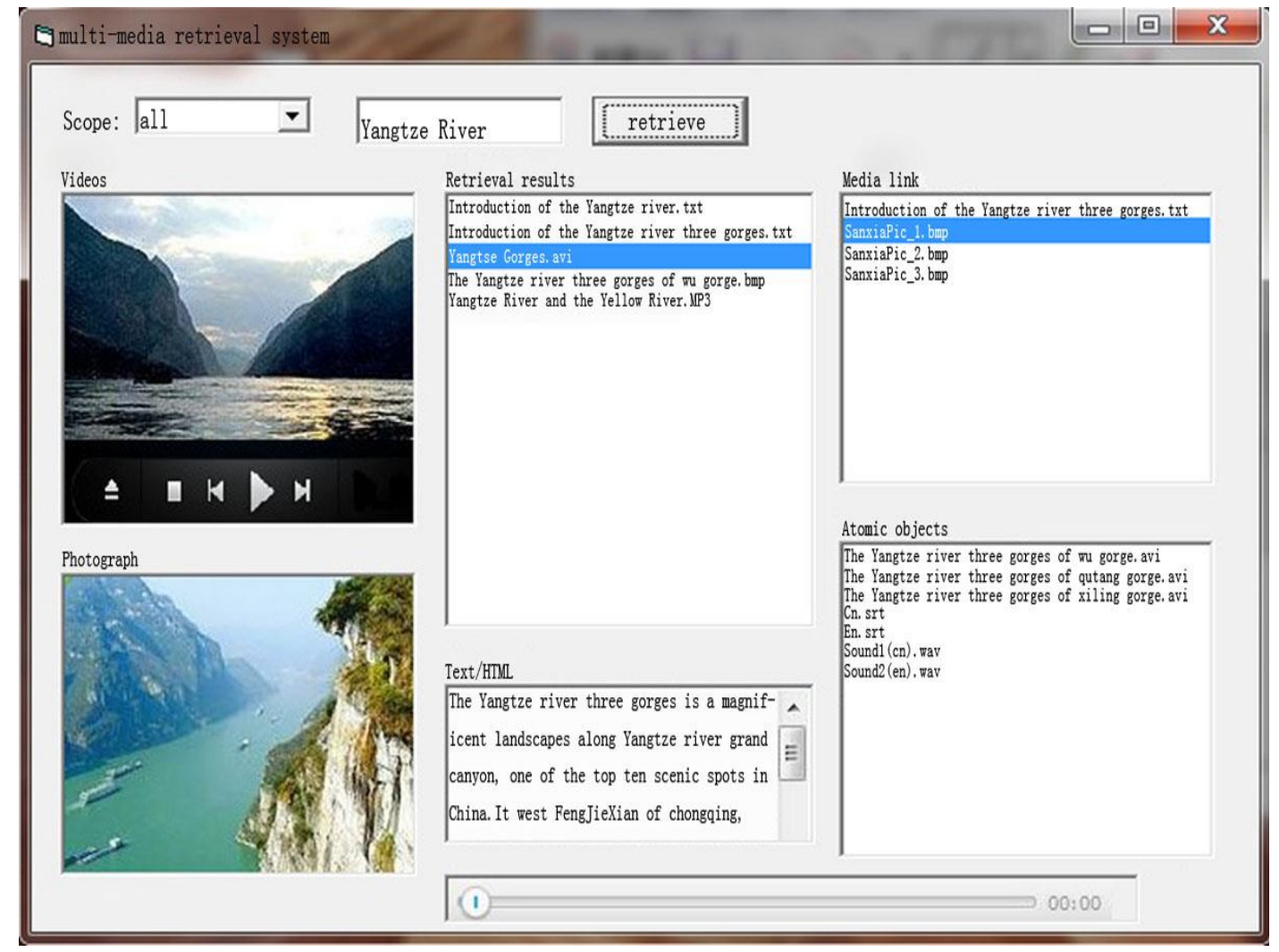

(1) Figure 8. Multi-media Retrieve

\section{Conclusion}

Multimedia data organization methods and storage structure are the key technology affecting the development of the digital library. This designs a novel data model, and efficiently realizing the organization of multi-media data. The innovation point expresses as:

1) It provides different fine granularity control on the media content, and sharply reduces the information redundancy within the media and among the medias, meanwhile better support the content-based retrieval.

2 ) It enhances the ability of abstraction and generalization of the model, which can support a variety of media objects, studio, or the performance of the query.

\section{References}

[1] J. Tu and P. Cao, "Research on Semantic Retrieval Model Based on Ontology in Digital Library", Journal of Intelligence, vol. 31, no. 7, (2012), pp.191-194.

[2] W. Z. Zhang, H. L. Zhang, X. Xu and H. He, "Distributed Search Engine System Productivity Modeling and Evaluation”, Journal of Software, vol. 23, no. 2, (2012), pp. 253-265. 
[3] M. M. Hu, Y. Tang, J. Li, "A Method for Distributed Spatial Metadata Retrieval Based on Directory Services. Computer Engineering and Science", vol. 33, no. 8, (2011), pp. 162-166.

[4] R. M. Bolle, B. L. Yeo and M. M. Yeung, "Video query: Research directions", IBM Journal of Research and Development, vol. 42, no. 2, (1998), pp. 233-252.

[5] R. Brunelli, O. Mich and C. M. Modena, "A Survey On The Automatic Indexing of Video Data", Journal of Visual Communication and Image Representation, vol.10, no. 2, (1999), pp. 78-112.

[6] Y. Jianfeng, Z. Yang and L. Zhanhuai, "A Multimedia Document Database Model Based on Multilayered Description Supporting Complex Multimedia Structural and Semantic Contents", Proceedings of the 10th International Multimedia Modelling Conference, (2004) January 5-7, Melbourne, Australia.

[7] G. Lu, "Multimedia Database Management Systems", Artech House Publishers, London and Boston, (1999).

[8] H. Chen, F. Li and S. Xu, "Modeling Multimedia and Multimedia Data", Computer Applications and Software, no.1, (2002), pp. 49-52.

[9] R. Weiss, A. Duda and D. K. Gifford, "Composition and Search with A Video Algebra", IEEE Multinedia, vol. 2, no. 1, (1995), pp. 12-25.

[10] H. Jiang, D. Montesi and A. K. Elmagarmid, "Integrated Video and Text for Content-Based Access to Video Databases", Multimedia Tools and Applications, vol. 9, no. 3, (1999), pp. 227-249.

[11] V. Oria, M. T. Ozsu, B. Xu, L. I. Cheng and P. Iglinski, "Xisual MOQL: The DISIMA visual query language", IEEE International Conference on Multimedia Computing and Systems (1999) June, Florence, Italy.

[12] R. Tusch, H. Kosch and L. Boszormenyi, "VideX: An integrated generic video indexing approach", Proceedings of The ACM Multimedia Conference, (2000) October, Los Angeles, USA.

[13] H. Kosch, R. Tusch, L. Boszormenyi, A. Bachlechner B. Doringer, C. Hofbauer, C. Riedler, M. Lang and C. Hanin, "SMOOTH - A Distributed Multimedia Database System", Rroceedings of the International VLDB Conference, (2001) September, Rome, Italy.

[14] F. Mohamed, M. Nordin, A. Rahman, Y. MLazim and S. B Mohamed, "Managing Multimedia Data: A Temporal-Based Approach. International Jounal of Multtimedia and Ubiquitous Engineering”, vol. 7, no. 4, (2012), pp. 73-86.

[15] L. Lin, "A Data Model with Multimeda Retrieval", Kroceedings of The 2nd International Conference on Computer, Information and Appiication, (2014) April, Angeles City (Clark), Philippines.

[16] L. Torresani, M. Szummer and A. Fitzgibbon, "Classemes: A Compact Image Descriptor for Efficient NovelClass Recognition and Search", Registration and Recognition in Images and Videos, vol. 532, (2014), pp. 95-111.

[17] Z. Li, Z. Zhou and D Hu, Scene Classification Using A Multi-Resolution Bag-of-Features Model”, Pattern Recognition, vol. 46, no 1, (2013), pp 424-433.

[18] S. Branson, C Wah, F. Schroff, B. Bahehko, P. Welinder, P. Perona and S. Belongie, "Visual Recognition With Humars In The Loon", Proceedings of The 11th European Conference on Computer Vision, (2010) September, Crete, Greece.

[19] D. Parikh and K. Grauman, "Relative Attributes", Proceedings of The 13th International Conference on Computer Vision, (2011) November, Barcelona, Spain.

[20] D. Parikh and K. Grauman, "Interactively Building a Discriminative Vocabulary of Nameable Attributes", Proceedings of The 2011 IEEE Conference on Computer Vision and Pattern Recognition, (2011) June; Colorado Springs, USA.

[21] S. Hwang, F Sha and K. Grauman, "Sharing Features between Objects and Their Attributes. Proceedings of The 201 TEEE Conference on Computer Vision and Pattern Recognition", (2011) June, Colorado Springs, USA

[22] . Aníbal Arias and J. Alfredo Sánchez, "Content-Based Search and Annotations in Multimedia Digital Loraries", Proceedings of the Fourth Mexican International Conference on Computer Science, (2003) September, Tlaxcala, Mexico.

[23] Z. Y. Cao and Y. Liu, "An Object-oriented Spatio-temporal Data Model”, Acta Geodaetica et Cartographica Sinica, vol. 31, no. 1, (2002), pp. 87-92.

[24] J. Su, Y. Z. Zhong and Q. Pu, "Study on the Layering Multimedia Database and Its Application", MicroComputer System, vol. 17, no. 2, (1996), pp. 28-33.

[25] R. Helm, R. Johnson, E. Gamma and J. Vlissides, "Design Patterns: Elements of Reusable Object-Oriented Software", Addison-Wesley, Boston (1994). 


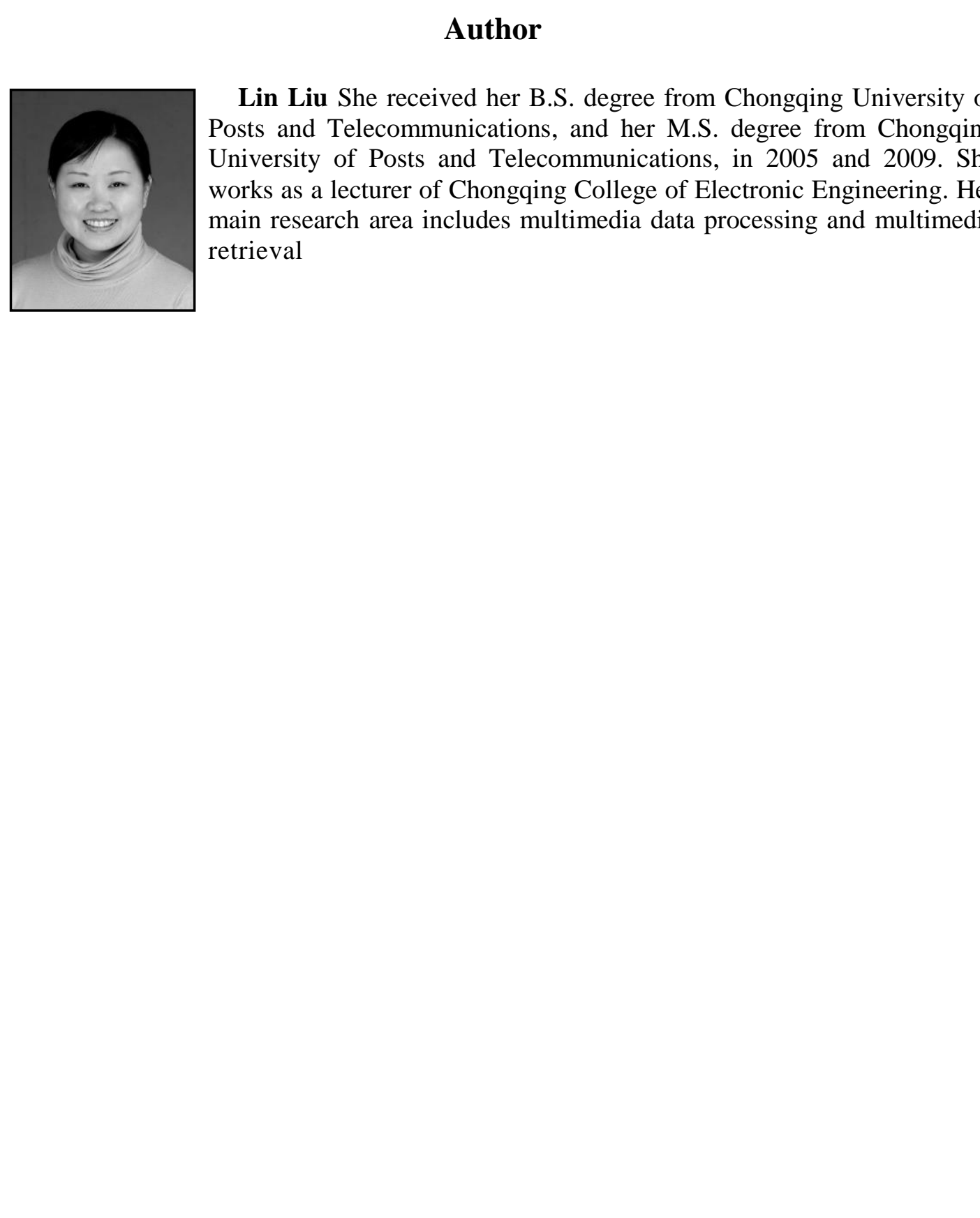


International Journal of Multimedia and Ubiquitous Engineering Vol.9, No.9 (2014)

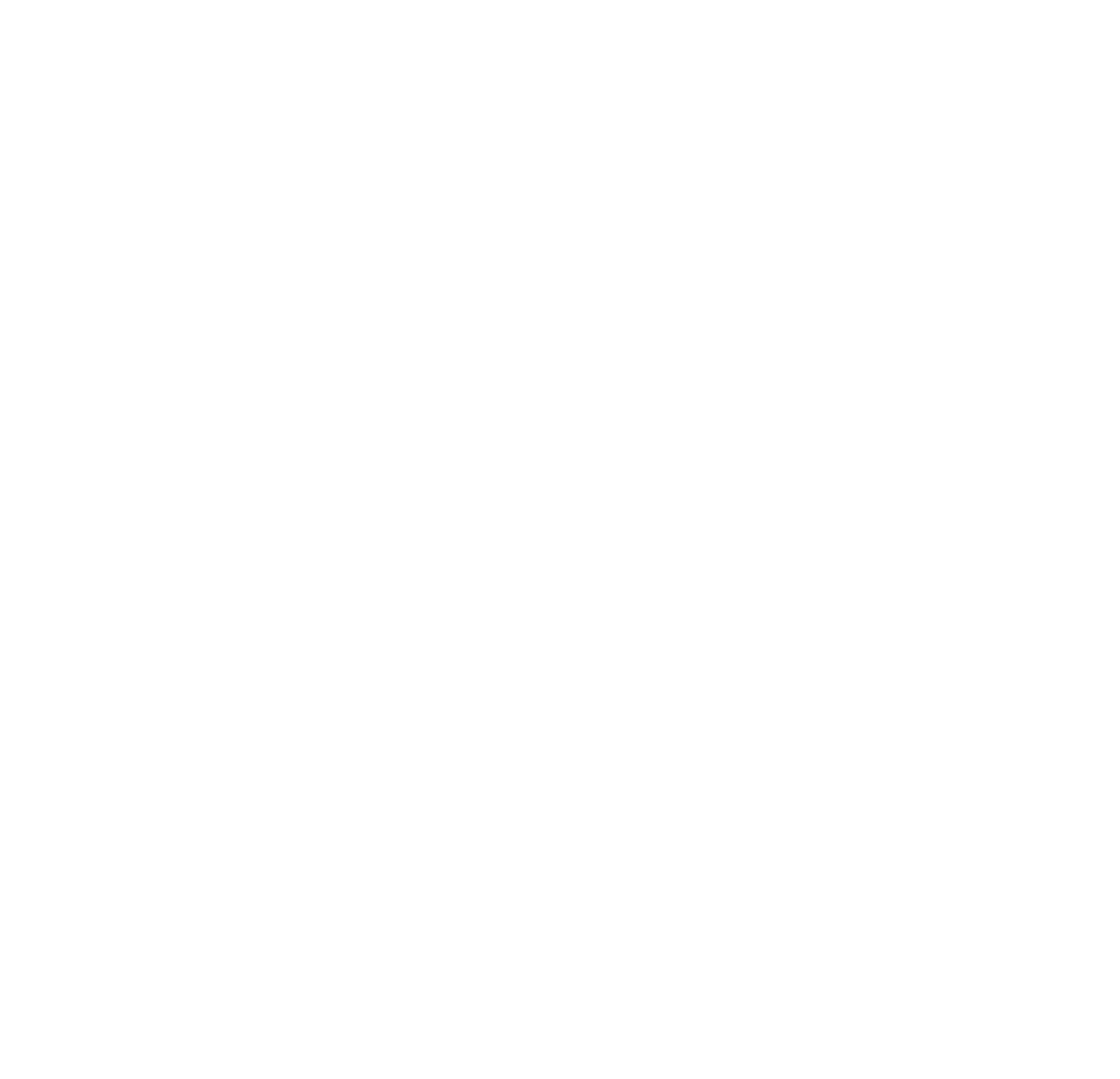

